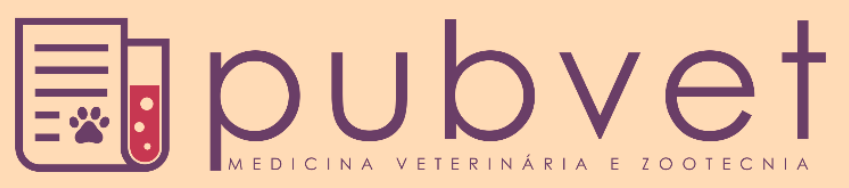

https://doi.org/10.31533/pubvet.v15n06a847.1-6

\title{
Acidente traumático por material perfurocortante em equino: Relato de caso
}

\author{
Marcela Lopes Ferraz ${ }^{1^{*}} \bullet$, Luis Henrique Silveira Baioco ${ }^{2}$, Liomara Andressa do Amaral \\ Kwirant $^{30}$, Rodrigo Berger Baptista ${ }^{4}$, Carolina Parisotto de Almeida ${ }^{\circ}$ \\ ${ }^{I}$ Graduanda em Medicina Veterinária, Centro de Ensino Superior dos Campos Gerais (CESCAGE). Ponta Grossa - PR. Brasil \\ ${ }^{2}$ Graduando em Medicina Veterinária, Centro de Ensino Superior dos Campos Gerais (CESCAGE). Ponta Grossa - PR. Brasil \\ ${ }^{3}$ Professora de Medicina Veterinária, Centro de Ensino Superior dos Campos Gerais (CESCAGE). Ponta Grossa - PR. Brasil \\ ${ }^{4}$ Médico Veterinário, Centro de Ensino Superior dos Campos Gerais (CESCAGE). Ponta Grossa - PR. Brasil \\ ${ }^{5}$ Médica Veterinária, Centro Universitário Fundação Assis Gurgacz (FAG). Cascavel - PR. Brasil \\ *Autora para correspondência, E-mail: marcelalopesferraz@hotmail.com
}

Resumo. De todas as espécies que necessitam de atendimento veterinário, os equinos são animais mais propensos a ferimentos, em função do tipo da área de atuação que são submetidos e o temperamento que apresentam. Devido a sua versatilidade é importante considerar cuidados básicos como o manejo e a aplicação dos conceitos da etologia com base no bem-estar específico do mesmo, em casos de ferimentos a posição clínica em relação a estes, deve ser criteriosamente analisado, avaliando as estruturas envolvidas, necessidade controle da hemorragia, sutura e inspeção minuciosa do animal juntamente com um exame clínico completo, afim de selecionar um tratamento adequado para a determinado caso, com o objetivo de uma cicatrização boa. Foi realizado um atendimento na cidade de Ponta Grossa, Paraná, sendo solicitado atendimento urgente do médico veterinário, por conta de um acidente com cerca de segurança serpentina e situação de hemorragia intensa, foram realizados os devidos procedimentos clínicos condizentes com o caso e gravidade do ferimento. $\mathrm{O}$ animal apresentou recuperação satisfatória e atualmente dispõe de ótimo estado de saúde sendo utilizado normalmente em sua área esportiva.

Palavras-chave: Clínica, equino, ferimentos, manejo

\section{Traumatic accident caused by sharps in horses: Case report}

Abstract. Of all the species that require veterinary care, horses are more prone to injuries, depending on the type of area they are subjected to and the temperament they present. Due to its versatility, it is important to consider basic care such as the management and application of the concepts of ethology based on the specific well-being of the same, in cases of injuries, the clinical position in relation to these must be carefully analyzed, evaluating the structures involved., need to control hemorrhage, suture and thorough inspection of the animal together with a complete clinical examination, in order to select an appropriate treatment for a given case, with the aim of good healing. Care was provided in the city of Ponta Grossa, Paraná, urgent care was requested from the veterinarian, due to an accident with serpentine safety and a situation of heavy bleeding, due clinical procedures were carried out consistent with the case and severity of the injury. The animal showed satisfactory recovery and currently has an excellent state of health being used normally in its sports area.

Keywords: Clinic, equine, management, wounds 


\section{Accidente traumático causado por objetos punzantes en caballos: Reporte de caso}

Resumen. De todas las especies que requieren atención veterinaria, los caballos son más propensos a lesionarse, según el tipo de área a la que estén sometidos y el temperamento que exhiban. Debido a su versatilidad, es importante considerar cuidados básicos como el manejo y aplicación de los conceptos de etología basados en el bienestar específico de los mismos. En los casos de lesión, se debe analizar cuidadosamente la posición clínica en relación a estos evaluando las estructuras involucradas, necesidad de control de hemorragias, sutura e inspección minuciosa del animal junto con un examen clínico completo, con el fin de seleccionar un tratamiento adecuado para un caso dado, con el objetivo de una buena cicatrización. Se brindó atención en la ciudad de Ponta Grossa, Paraná, se solicitó atención urgente al veterinario, debido a un accidente con seguridad serpentina y una situación de sangrado abundante, se realizaron los procedimientos clínicos debidos de acuerdo al caso y gravedad de la lesión. El animal mostró una recuperación satisfactoria y actualmente tiene un excelente estado de salud siendo utilizado con normalidad en su área deportiva.

Palabras clave: Clínica, equinos, lesiones, manejo

\section{Introdução}

O cavalo é um animal versátil e em todo Brasil pode ser utilizado em diferentes atividades, como alguns esportes mais clássicos, salto e adestramento e outros mais regionais, como a vaquejada e prova de três tambores (Santos et al., 2017). Também existe o uso militar, praticado em diferentes estados do país, usos agropecuários e de trabalho (Lima et al., 2006). Se os cavalos são usados para o prazer, trabalho, ensino e pesquisas, um nível adequado de cuidados com os animais deve ser proporcionado e implementado, com corretas práticas de manejo e aplicação dos conceitos da etologia, com base no bemestar específico da espécie (Malinowski et al., 2006). De todas as espécies de animais domésticos, os cavalos são particularmente propensos a ferimentos (Thomassian, 2005). As condições às quais os cavalos são submetidos, o tipo de trabalho que realizam e em que estão envolvidos e o seu temperamento natural são provavelmente as razões para uma incidência tão elevada de ferimentos. Os objetivos no manejo desses ferimentos são a identificação de todas as estruturas envolvidas, controle da hemorragia e avaliação completa (Pollock \& Schumacher, 2012). Um exame clínico completo, mas breve, deve ser realizado antes de dar início ao tratamento. Se a ferida está localizada em um membro, a presença e o grau de claudicação podem ser avaliados como indicadores de ferimentos potencialmente mais graves. Feridas localizadas em cima ou próximo de articulações, bainhas tendinosas ou tendões (particularmente tendões flexores) e aquelas que expõem ou penetram o osso, devem ser exploradas meticulosamente. Se há hemorragia grave, o controle desta, deve ser realizado antes de qualquer outro procedimento (Hardy \& Wilkins, 2005). O tratamento de escolha terá como base os aspectos do ferimento e seu tipo, sendo avaliados critérios essenciais visando a necessidade de sutura, colocação de drenos, desbridamento, limpeza intensiva, lavagens articulares, entre outros métodos. Além disso, deve-se avaliar a possibilidade de cicatrização do ferimento em questão.

O presente relato tem como objetivo descrever um caso de acidente traumático por objeto perfurocortante em uma égua crioula ocorrido na cidade de Ponta Grossa, região dos campos gerais no Paraná.

\section{Relato de caso}

Uma égua da raça Crioula, com cinco anos de idade mantida na cocheira durante a noite e solta em piquete durante o dia, foi atendida devido a um acidente causado por cerca de segurança (serpentina). $\mathrm{O}$ proprietário relatou que a égua havia escapado do seu piquete habitual, se assustou e acabou se enroscando na cerca, lesionando a região ventral do pescoço. Durante o atendimento constatou-se que houveram rupturas dos músculos esternocefálico e braquiocefálico, além da completa ruptura da veia jugular esquerda, o que ocasionou uma hemorragia. O responsável pelo animal havia pinçado a veia 
afim de diminuir a hemorragia e também fez uso de pó de café na região com a mesma finalidade, não obtendo sucesso na utilização do pó de café. A paciente em questão estava com as vacinações em dia, mesmo assim realizou-se a profilaxia antitetânica em dose de 10.000 UI de soro antitetânico por via intramuscular associado ao anti-inflamatório, sendo o medicamento de eleição flunixina meglumina em dose de $10 \mathrm{~mL}$ por via intravenosa.

No primeiro momento após a anamnese descrita pelo proprietário, foi realizado um exame físico geral do animal, aferindo os sinais vitais: a frequência cardíaca foi de $32 \mathrm{bpm}$, a frequência respiratória foi de $18 \mathrm{rpm}$, as mucosas estavam normocoradas e o TPC foi de dois segundos. Após exame clínico realizou-se o pinçamento correto da veia jugular externa com o auxílio de uma pinça Kelly e posteriormente foi feita a tricotomia e lavagem do ferimento, antissepsia com iodo povidine tópico $10 \%$, enxágue com água corrente e secagem com papel toalha. Após a lavagem o médico veterinário obstruiu a veia jugular com fio de nylon 5-0 afim de realizar a dissecação da mesma, dados três nós simples no mesmo, evitando a possibilidade de rompimento.

Para que se realizasse o procedimento de forma confortável e indolor utilizou-se da técnica de anestesia local com a lidocaína + xilazina, feitos botões de $0,03 \mathrm{mg} / \mathrm{kg}$ ao redor do ferimento, como o paciente era extremamente tranquilo, realizou-se sedação com xilazina $0,03 \mathrm{mg} / \mathrm{kg}$. Em função da reestruturação do tecido lesado, e da orientação do corte, fez-se desbridamento da região e a sutura padrão sultan na musculatura, para que não houvesse laceração das fibras musculares. O fio utilizado foi o poligalactina 2.0, conforme a necessidade de absorção pelos tecidos.

Considerando a perda tecidual tanto cutânea como muscular e a exsudação presente no ferimento, optou-se pela colocação de um dreno para impedir o acúmulo de líquido. A fixação do dreno foi realizada com sutura em sultan sendo utilizado fio de nylon 1-0. Após o término de todas as suturas, foi feito a limpeza do local com fitoclean e aplicação de spray prata sobre a região, com finalidade de proteção contra moscas de miíase (Figura 1). Administrou-se 101 de ringuer lactato e 11 de bioxan, visando auxiliar na reidratação e recomposição dos eletrólitos, em razão da hemorragia.

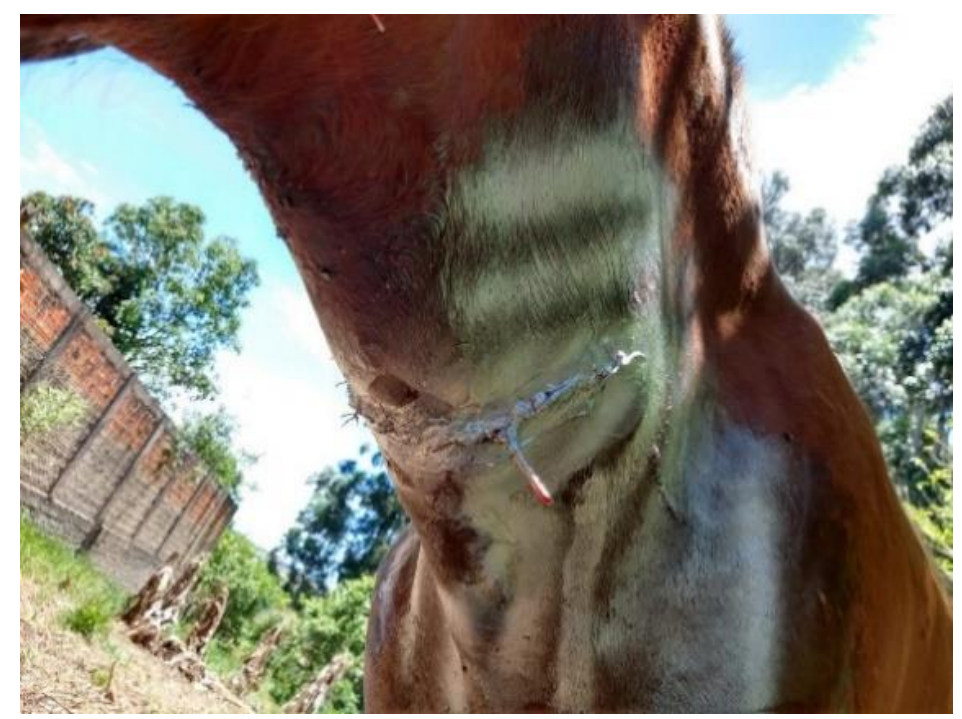

Figura 1. Ferimento suturado com presença de dreno.

Em relação à cicatrização, como não foi possível o fechamento total da ferida, elegeu-se a possibilidade de cicatrização por $3^{\mathrm{a}}$ intenção, com cuidados diários e acompanhamento da resposta do tratamento (Figura 2B). As prescrições feitas após o tratamento emergencial, foram benzilpenicilina potássica $30 \mathrm{~mL}$ a cada 12 horas por via intramuscular durante seis dias, lavagem da ferida com água associada a barbatimão (Stryphnodendron adstringens) fervida diariamente até a cicatrização completa e aplicação do fitoclean spray para auxiliar na limpeza e por fim o spray prata. Os pontos foram retirados após sete dias, e alguns já haviam caído ou ficaram mais soltos, depois da retirada, continuou-se o 
acompanhamento diário, até o total fechamento do ferimento, o processo de cicatrização completa durou 30 dias.

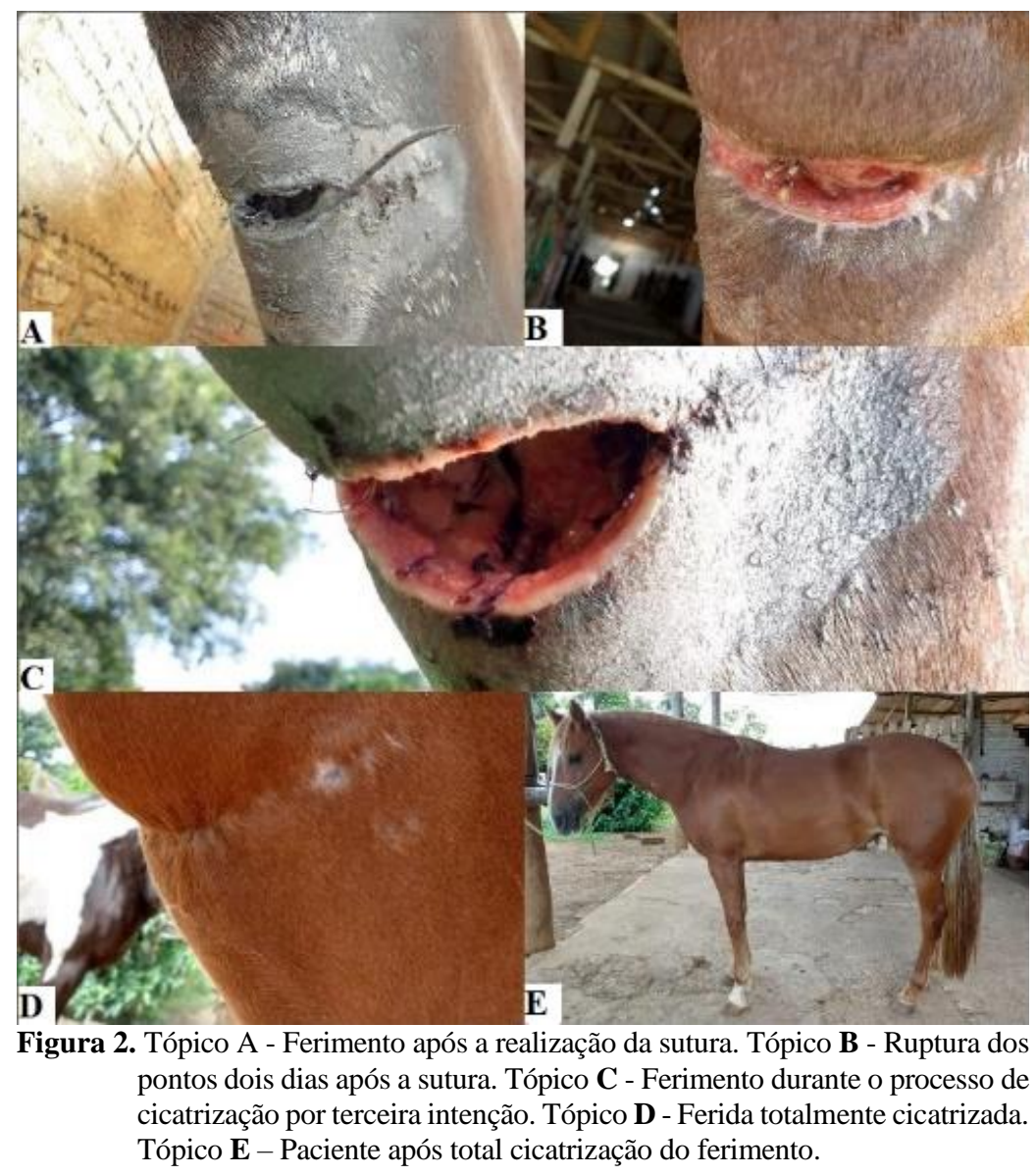

\section{Discussão}

De acordo com Pessoa et al. (2014), feridas de origem traumática são as lesões mais prevalentes nos equinos, distinguindo-se daquelas em outras espécies devido a características únicas, incluindo taxas de cicatrização diferentes entre raças, neste caso a lesão ocorreu devido a um acidente com material perfurocortante, que exigiu maiores cuidados durante o processo de cicatrização devido à gravidade do ferimento.

A fase inflamatória é essencial para a proteção contra a infecção, bem como para iniciar o processo de reparação. Atribuiu-se aos macrófagos um papel essencial na transição entre a inflamação e a reparação, pela liberação de diversos fatores de crescimento e citocinas (Latimer et al., 1992). Neutrófilos são as primeiras células a chegar à ferida, com maior concentração 24 horas após a lesão. São atraídos por substâncias quimiotáticas liberadas por plaquetas. Os neutrófilos aderem à parede do endotélio mediante ligação com as selectinas (receptores de membrana). Neutrófilos produzem radicais livres que auxiliam na destruição bacteriana e são gradativamente substituídos por macrófagos (Broughton et al., 2006). Considerando tais colocações, foi possível avaliar que a higiene e antissepsia realizada no ferimento durante as fases de cicatrização, foram essenciais para que houvesse um estímulo aos mediadores cicatrizantes, não apresentando maiores complicações que exigissem uma nova avaliação e protocolo medicamentoso. Tazima et al. (2008) relatam que reparo de feridas, uma solução de continuidade dos tecidos, decorrente da lesão por agentes mecânicos, térmicos, químicos e bacterianos, é o esforço dos tecidos para restaurar a função e estruturas normais, que apresentam fases características do processo. A fase proliferativa caracterizada pela formação de tecido de granulação, inicia-se por volta do $3^{\circ}$ dia após a lesão, perdura por duas a três semanas e é o marco inicial da formação 
da cicatriz. Foi possível observar de maneira nítida este processo durante a recuperação do paciente, que mostrou os primeiros sinais de cicatriz na segunda semana após a retirada dos pontos.

A contração geralmente inicia-se em feridas profundas na segunda semana após a lesão, uma vez que neste período, encontra-se densamente povoada por fibroblastos e pode continuar por várias semanas, reduzindo a área de superfície da ferida original em 40 a 80\% (Di Pietro \& Burns, 2003). A presença de tecido de granulação é notada dentro de três a cinco dias, sendo formado a partir da proliferação capilar e de fibroblastos, o que o torna friável; porém, resistente à infecção (Hardy \& Wilkins, 2005). Durante o acompanhamento e avaliação do processo, optou-se pela cicatrização por $3^{\text {a }}$ intenção devido a deiscência dos pontos e amplitude da ferida, conforme Broughton et al. (2006) o sucesso da cicatrização está relacionado ao equilíbrio entre a síntese da nova matriz e a lise da matriz antiga, sendo melhor quando a deposição de colágeno é maior.

Considerando que os equinos também produzem níveis menores de outros mediadores necessários para reforçar a resposta inflamatória e para induzir a contração da ferida (Swaim et al., 1996) associouse ao uso dos medicamentos a água fervida de barbatimão, durante 25 dias, apresentando resultados visíveis nos quatros primeiros dias, melhorando o aspecto do ferimento e demonstrando cicatrização satisfatória sem complicações.

Na medicina popular, o barbatimão, principalmente na forma de extrato da casca é utilizado para o tratamento de leucorreia, diarreia, hemorragia, hemorroida, feridas, conjuntivite, inflamação da garganta, corrimento vaginal e úlcera gástrica (Costa et al., 2012). No entanto, entre os principais efeitos medicinais atribuídos a esse fitoterápico destacam-se a sua propriedade cicatrizante (Eurides et al., 1996; Costa et al., 2012; Eurides et al., 2010; Hernandes et al., 2010; Lima et al., 2006), antimicrobiana (Costa et al., 2012), antiofídica (Lucena et al., 2009) e antioxidante (Souza et al., 2007).

Associado à técnica fitoterápica, optou-se pelo uso do Fitoclean spray, é uma solução higienizadora de ferimentos (serosa e mucosa) composta por plantas que promovem limpeza profunda e tem função adstringente (Organnact, 2020). Spray prata também foi utilizado logo após a aplicação, para evitar a possibilidade de miíase.

\section{Conclusões}

Considerando os acidentes traumáticos em equinos, é de extrema importância possuir conhecimento de manejo e estrutura para criação da espécie, em casos emergenciais o atendimento do médico veterinário é indispensável para que não ocorra a perda do animal. Com o avanço das terapias alternativas e sua eficácia, a recuperação de lesões e diminuição de complicações ou doenças secundárias, tornou-se algo mais comum, diminuindo a possibilidade de mortalidade do animal acometido e garantindo uma vida saudável posteriormente. Sendo assim o cuidado e acompanhamento do paciente é essencial afim de obter-se recuperação satisfatória.

\section{Referências}

Broughton, G., Janis, J. E., \& Attinger, C. E. (2006). The basic science of wound healing. Plastic and Reconstructive Surgery, 117(7S), 12S-34S. https://doi.org/10.1097/01.prs.0000225430.42531.c2.

Costa, J. G. M., Leite, G. O., Dubois, A. F., Seeger, R. L., Boligon, A. A., Athayde, M. L., Campos, A. R., \& Rocha, J. B. T. (2012). Antioxidant effect of Stryphnodendron rotundifolium Martius extracts from Cariri-Ceará state (Brazil): Potential involvement in its therapeutic use. Molecules, 17(1), 934950. https://doi.org/10.3390/molecules17010934.

Di Pietro, L. A., \& Burns, A. L. (2003). Wound healing: methods and protocols (Vol. 78). Springer.

Eurides, D., Frando, L. G., Moura, M. I., Campos, S. B. S., \& Freitas, S. L. R. (2010). Stryphnodendron adstringens (Martius) coville. In L. A. F. Silva, D. Eurides, J. R. Paula, \& M. I. Moura (Eds.), Manual do barbatimão (pp. 69-78). Kelps.

Hardy, J., \& Wilkins, P. A. (2005). Equine emergency medicine: Wounds and lacerations. In C. M. Kahn (Ed.), The merck veterinary manual (pp. 1441-1433). Merial.

Hernandes, L., Pereira, L. M. S., Palazzo, F., \& Mello, J. C. P. (2010). Wound-healing evaluation of ointment from Stryphnodendron adstringens (barbatimão) in rat skin. Brazilian Journal of 
Pharmaceutical Sciences, 46(3), 431-436.

Latimer, K. S., Meyer, D. J., \& Ettinger, S. J. (1992). Os leucócitos na saúde e na moléstia. In S. J. Ettinger (Ed.), Tratado de Medicina Interna Veterinária (Vol. 4, pp. 2616-2664). Manole.

Lima, R. A. S., Shirota, R., \& Barros, G. S. C. (2006). Estudo do complexo do agronegócio cavalo. Confederação da Agricultura e Pecuária do Brasil.

Lucena, M. N., Mendes, M. M., \& Brandeburgo, M. I. H. (2009). Avaliação da estabilidade da pomada à base de Stryphnodendron adstringens (Mart.) Conville e sua eficácia na neutralização dos efeitos locais induzidos pela peçonha de Bothrops pauloensis. Horizonte Científico, 3(1), 1-29.

Malinowski, K., Shock, E. J., Rochelle, P., Kearns, C. F., Guirnalda, P. D., \& McKeever, K. H. (2006). Plasma $\beta$-endorphin, cortisol and immune responses to acute exercise are altered by age and exercise training in horses. Equine Veterinary Journal, 38(S36), 267-273. https://doi.org/10.1111/j.20423306.2006.tb05551.x.

Organnact. Linha Dermatológicos: Fitoclean Spray. In: Fitoclean. [S. 1.], 9 jul. (2020). Disponível em: https://www.organnact.com.br/produto/linha-dermatologicos/fitoclean/. Acesso em: 25 jun. 2020.

Pessoa, A. F. A., Pessoa, C. R. M., Miranda Neto, E. G., Dantas, A. F. M., \& Riet-Correa, F. (2014). Doenças de pele em equídeos no semiárido brasileiro. Pesquisa Veterinária Brasileira, 34(8), 743748.

Pollock, P. J., \& Schumacher, J. (2012). Principles of wound management. Equine Medicine, Surgery and Reproduction.

Santos, B. E. S., Brandi, R. A., \& Gameiro, A. H. (2017). Estudo do mercado e produção do cavalo Brasileiro de hipismo no estado de São Paulo. PUBVET, 12(2), 1-11. https://doi.org/10.22256/pubvet.v12n2a35.1-11.

Souza, T. M., Severi, J. A., Silva, V. Y. A., Santos, E., \& Pietro, R. C. L. R. (2007). Bioprospecção de atividade antioxidante e antimicrobiana da casca de Stryphnodendron adstringens (Mart.) Coville (Leguminosae-Mimosoidae). Revista de Ciências Farmacêuticas Básica e Aplicada, 28(2), 221-226.

Swaim, S. F., Vaughn, D. M., Kincaid, S. A., Morrison, N. E., Murray, S. S., Woodhead, M. A., Hoffman, C. E., Wright, J. C., \& Kammerman, J. R. (1996). Effect of locally injected medications on healing of pad wounds in dogs. American Journal of Veterinary Research, 57(3), 394-399.

Tazima, M. F. G. S., Vicente, Y. A. M. V. A., \& Moriya, T. (2008). Wound biology and healing. Medicina, 41(3), 259-264.

Thomassian, A. (2005). Enfermidades dos cavalos. Livraria Varela.

Histórico do artigo:

Recebido: 26 de janeiro de 2021

Aprovado: 25 de fevereiro de 2021.
Licenciamento: Este artigo é publicado na modalidade Acesso Aberto sob a licença Creative Commons Atribuição 4.0 (CC-BY 4.0), a qual permite uso irrestrito, distribuição, reprodução em qualquer meio, desde que o autor e a fonte sejam devidamente creditados. 